\title{
DERIVADOS HEMATOLÓGICOS: PLASMA RICO EN PLAQUETAS (PRP)
}

\author{
D. Poggio Cano 1,2, C. Álvarez Gómez 2,3, R. García Elvira ${ }^{1,2}$ \\ ${ }^{1}$ Unidad de Pie y Tobillo. Hospital Clínico y Provincial de Barcelona. Universitat de Barcelona \\ ${ }^{2}$ Hospital Sanitas CIMA. Barcelona \\ ${ }^{3}$ Unidad de Pie y Tobillo. Hospital de la Santa Creu i Sant Pau. Universitat Autònoma de Barcelona.
}

\section{Introducción}

El incremento en el uso de plasma rico en plaquetas (PRP) en el aparato locomotor ha generado un gran abanico de posibilidades y potenciales indicaciones, a la par que un gran número de interrogantes sobre las aplicaciones clínicas y las limitaciones de esta terapia en desarrollo.

El denominado PRP es un producto refinado de la sangre autóloga con un aumento de la concentración de plaquetas en relación con la sangre total y, consecuentemente, se trataría de un producto rico en factores biológicos, fundamentalmente factores de crecimiento (FC) contenidos mayoritariamente en las plaquetas y esenciales en la respuesta biológica del organismo en las lesiones o traumatismos. Existen en el mercado productos denominados plasma rico en FC (PRGF) atendiendo a esta gran concentración en FC y haciendo así énfasis en su importancia.

LoS PRP se preparan mediante separación de las distintas fracciones de la sangre con sistemas de centrifugado, existiendo a día de hoy más de 40 sistemas disponibles en el mercado a tal efecto(1).

Su uso se remonta a más de 50 años en el campo de la cirugía maxilofacial, si bien en el mundo de las lesiones musculoesqueléticas se extendió posteriormente ${ }^{(2)}$.

Hoy en día una búsqueda en Google nos permite obtener unos 400.000 resultados relacionados con los PRP y, en PubMed, una búsqueda genérica sobre PRP ofrece más de 5.000 resultados de artículos de peer-review.

Aunque la terapia con PRP es prometedora en diferentes áreas, parece razonable pensar que no es apropiada en todos los casos. Para delimitar su uso y sus indicaciones, se precisa aclarar y acabar de comprender en detalle el proceso biológico de reparación y con ello las variables que pueden afectar a la efectividad del PRP.

FS $\odot 2017$ SEMCPT. Publicado por Imaidea Interactiva en FONDOSCIENCE ${ }^{\circledR}$ (www.fondoscience.com). 
La mayor parte de los expertos en la materia recalcan en sus trabajos la importancia de la composición del PRP. Es necesario ajustar y clasificar esta composición y con ello el producto final, en función del efecto buscado y la patología tratada.

\section{Técnica de obtención y clasificación}

La literatura acerca del uso de los PRP en la clínica sugiere una considerable variabilidad en su efectividad incluso en escenarios clínicos similares. Las razones para esta variabilidad de resultados no están del todo dilucidadas, pero se barajan varios motivos. Uno de estos motivos es que la composición de los PRP varía en función de la técnica utilizada ${ }^{(3)}$.

Aunque todos los preparados de PRP contienen un grupo básico de FC, la concentración relativa de cada factor varía entre diferentes preparaciones, alterando su efectividad clínica para aplicaciones específicas.

El PRP se puede obtener mediante kits desechables donde todo el procesado es cerrado o bien puede obtenerse de forma manual, mediante pipeteo.

En el caso de la obtención mediante kits desechables, salvando pequeñas variaciones, los pasos serían los que se detallan a continuación.

Extraer la sangre del paciente y disponerla en tubos aditivados y aptos para introducir en una centrifugadora concreta. La sangre debe centrifugarse inmediatamente tras la extracción y sin haber sido refrigerada; equilibrar la centrífuga y hacer girar la sangre estableciendo los parámetros (velocidad y tiempo) según lo propuesto por el fabricante; extraer las fracciones adecuadas según lo indicado; por último, extraer el PRP siguiendo las normas del proveedor (resuspender las plaquetas y extraer PRP con la jeringa adecuada). Toda la manipulación de los dispositivos debe realizarse bajo condiciones asépticas, siguiendo los protocolos de asepsia de cada centro de trabajo, para minimizar las posibilidades de contaminar las fracciones de plasma obtenidas.

En la "técnica abierta" de obtención, se precisa una técnica de pipeteo. Con ella, a partir de menores volúmenes de sangre y un equipamiento sencillo, se obtiene una mayor concentración de $F C^{(4)}$.

Las fracciones con mayor contenido de plaquetas son las que se encuentran inmediatamen- te por encima de la serie blanca. De ella se obtiene la fracción de PRGF, donde se encuentra un concentrado en plaquetas hasta 5 veces mayor que en sangre periférica(5).

Dada la falta de estudios bien diseñados sobre la estabilidad de los distintos componentes del PRP, se considera que entre la extracción de la sangre y la administración del PRP (ya preparado) no deberían pasar más de 45 minutos $^{(6)}$.

Cuando el PRP se destina a tratar lesiones de partes blandas, no se considera necesaria la activación previa, debido a que esta se produce in situ al contacto con el colágeno tendinoso o con el propio coágulo de la rotura fibrilar.

Cuando el PRP se utiliza para facilitar la osteointegración de implantes o cuando se usa para el tratamiento de la osteoartritis de rodilla suele preferirse cierta activación (con trombina o cloruro cálcico) que, además, le puede conferir una consistencia gelatinosa que facilita su uso quirúrgico(7).

Dependiendo del sistema de obtención, las concentraciones de plaquetas, leucocitos, eritrocitos y FC pueden variar. Hay varios métodos comercializados en la actualidad para la preparación de PRP que presentan distintas composiciones mediante el aditamento del PRP con diferentes proporciones de leucocitos durante su procesamiento.

Al utilizar los distintos métodos se obtienen diferentes fracciones y podemos encontrar distintas denominaciones en la literatura, como: preparado rico en factores de crecimiento (PRGF), plasma rico en plaquetas y factores de crecimiento (PRPGF) -Tecnología Endoret ${ }^{\circledR}\left(\mathrm{PRGF}^{\circledR}\right)(\mathrm{BTI})$-, plasma rico en plaquetas (PRP), plasma pobre en plaquetas (PPP), plasma rico en plaquetas y rico en leucocitos (LR-PRP) -GPS ${ }^{\circledR}$ III (Biomet)-, plasma rico en plaquetas y pobre en leucocitos (LP-PRP) -Cascade ${ }^{\circledR}$ PRP System (MTF), ACP (Arthrex)-. Todo ello pone de manifiesto la gran variabilidad existente en los preparados denominados genéricamente $\mathrm{PRP} \mathrm{P}^{(8)}$.

Sobre los distintos preparados de PRP se ha tratado de generar una clasificación de consenso. Esta clasificación distingue 4 grandes tipos de PRP, basándose en su contenido celular y su arquitectura de fibrina ${ }^{(9)}$.

- Pure platelet-rich plasma (P-PRP) (por ejemplo, PRGF-Endoret ${ }^{\circledR}$ Technique).

- Leukocyte and platelet-rich plasma (LPRP) (por ejemplo, Biomet GPS ${ }^{\circledR}$ System).

- Pure platelet-rich fibrin (P-PRF) (por ejemplo, Fibrinet $\left.{ }^{\circledR}\right)$. 
- Leukocyte and platelet-rich fibrin (L-PRF) (por ejemplo, IntraSpin L-PRF ${ }^{\circledR}$ ).

Estos 4 tipos de PRP presentan diferencias biológicas y diferentes aplicaciones (Tabla 1). Las diferencias en la composición cualitativa y cuantitativa de los distintos preparados de PRP es un factor importante en la dificultad para encontrar diferencias significativas acerca del uso de estos productos, mediante la medicina basada en la evidencia (MBE). Esto se ve corroborado por varios estudios que ponen de manifiesto el distinto efecto de unos y otros preparados de PRP.

En el estudio de Sundman et al.(10) comparan 2 preparados comerciales (GPS ${ }^{\circledR}$, Biomet, y ACP ${ }^{\circledR}$, Arthrex), objetivando distintas concentraciones de FC en relación con la ratio leucocitos/plaquetas.

Por otra parte, en el estudio de Drago et al. ${ }^{(11)}$ evalúan comparativamente 2 preparados de PRP. Por un lado, un preparado LP-PRP (Cascade ${ }^{\circledR}$ PRP System, MTF) y, por otro, uno LR-PRP (GPS ${ }^{\circledR}$ III, Biomet), viendo significativas diferencias en el estudio histológico. Mientras el LP-PRP no genera ninguna reacción inflamatoria en el tejido, en los especimenes tratados con LR-PRP se aprecia una intensa reacción inflamatoria aguda. Este hecho hace pensar en efectos biológicos e indicaciones distintas e incluso opuestas para ambos, según el efecto buscado.

\section{Evidencias de seguridad y consideraciones legales}

Los avances médicos comienzan a menudo su implantación antes de que exista una legislación que los contemple y que proporcione a pacientes y profesionales un marco jurídico que garantice un uso seguro y racional de los mismos. Existe una gran diferencia a nivel de regulación entre un tratamiento considerado medicamento, el cual debe pasar rígidos cauces y comisiones, y aquel producto considerado producto sanitario (PS).

La Agencia Española de Medicamentos y Productos Sanitarios (AEMPS) considera que el PRP es un medicamento de uso humano que se puede utilizar al amparo del artículo 5 de la Directiva 2001/83/CE, de 6 de noviembre, y las disposiciones legales que transponen dicha Directiva en nuestro país.

La AEMPS ha establecido qué requisitos mínimos ha de cumplir el PRP como medicamento de uso humano ${ }^{(12)}$. Sin embargo, los kits desechables cerrados empleados para su obtención, siguiendo el RD 1591/2009(13) y las directrices comunitarias, son considerados y tratados como PS ${ }^{(14)}$.

Los distintos estudios y la práctica muestran que la tolerancia a la infiltración es en general muy buena. El resultado del PRP es un producto autólogo que contiene una mezcla de FC sin potencial de respuesta inmunológica. Es razonable pensar que la naturaleza autóloga del PRP facilite su tolerancia terapéutica, pero tolerancia no es sinónimo de inocuidad.

El factor de crecimiento endotelial vascular (VEGF), el factor de crecimiento fibroblástico básico, el factor de crecimiento hepatocitario (HGF) y el factor de crecimiento insulínico son factores de especial relevancia en el crecimiento de ciertos tumores debido a su elevado potencial angiogénico. No obstante, estas propiedades de los FC, proclives a la generación y perpetuación de tumores, solo han sido descritas en animales de experimentación, sin evidencias, hasta el momento, que relacionen el uso de PRP en humanos con algún tipo de transformación carcinomatosa ${ }^{(15)}$.

\section{El proceso de reparación natural y el plasma rico en plaquetas}

El PRP debe su interés terapéutico al papel instrumental decisivo de las plaquetas en el proceso de curación y reparación de una herida tisular. El proceso de reparación del sistema musculoesquelético de forma genérica comienza con la formación del coágulo y la degranulación de las plaquetas, lo cual libera FC y citocinas. Este microambiente facilita la quimiotaxis y la proliferación celular local, ayudando en general a la formación del tejido de reparación más habitual, el tejido cicatricial fibroblástico ${ }^{(16)}$.

Se ha reportado que este microambiente de FC puede facilitar, durante la formación del callo óseo, la formación de hueso nuevo. Los FC encontrados en el coágulo natural son: transforming growth factor beta (TGF- $\beta$ ), platelet-derived growth factor (PDGF), insulin-like growth factor (IGF), VEGF, epidermal growth factor (EGF) y fibroblast growth factor-2 (FGF-2).

Algunos de estos factores han mostrado su capacidad de acelerar una o más fases del proceso osteogénico. Así el PDGF, el EGF y el FGF-2 podrían 


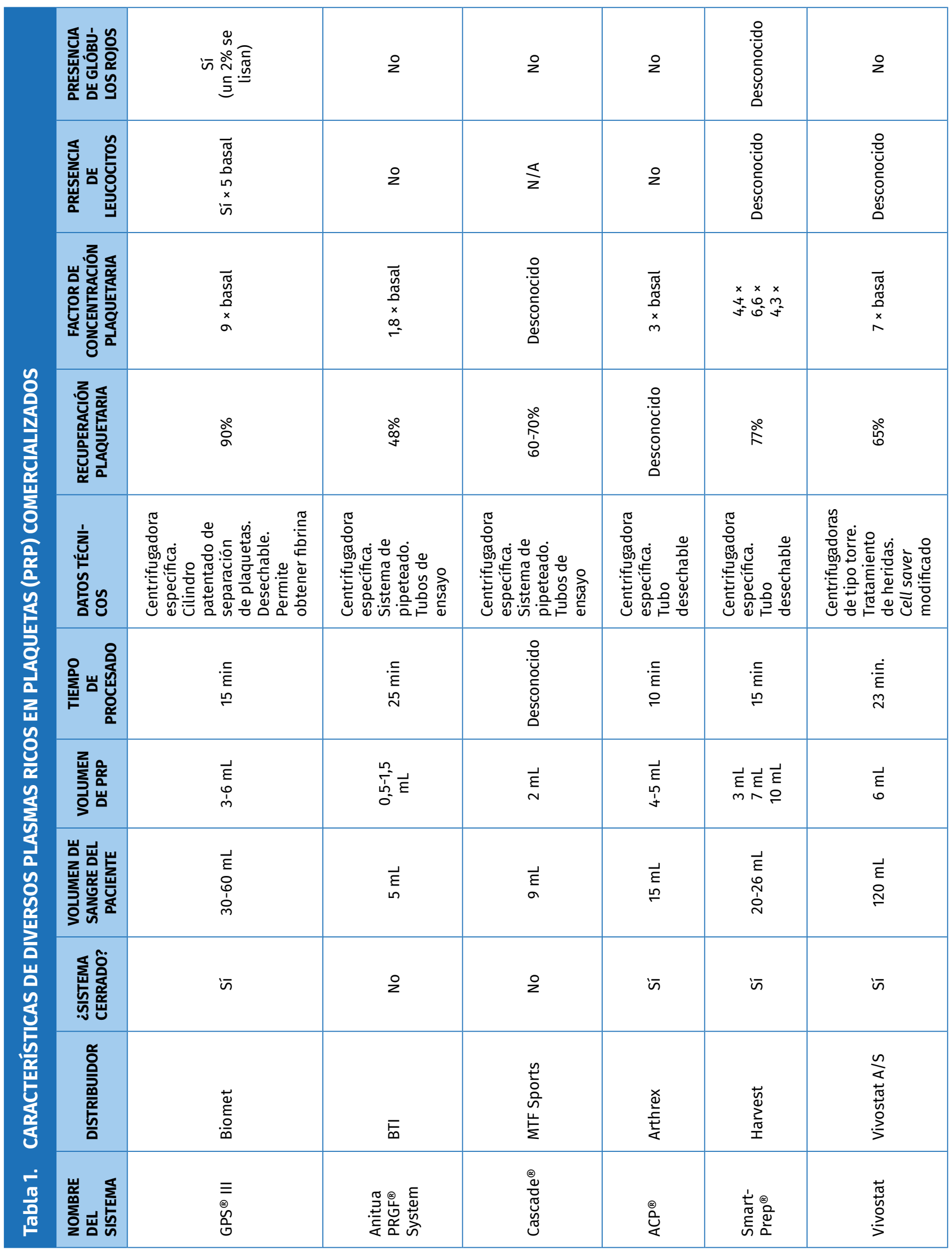




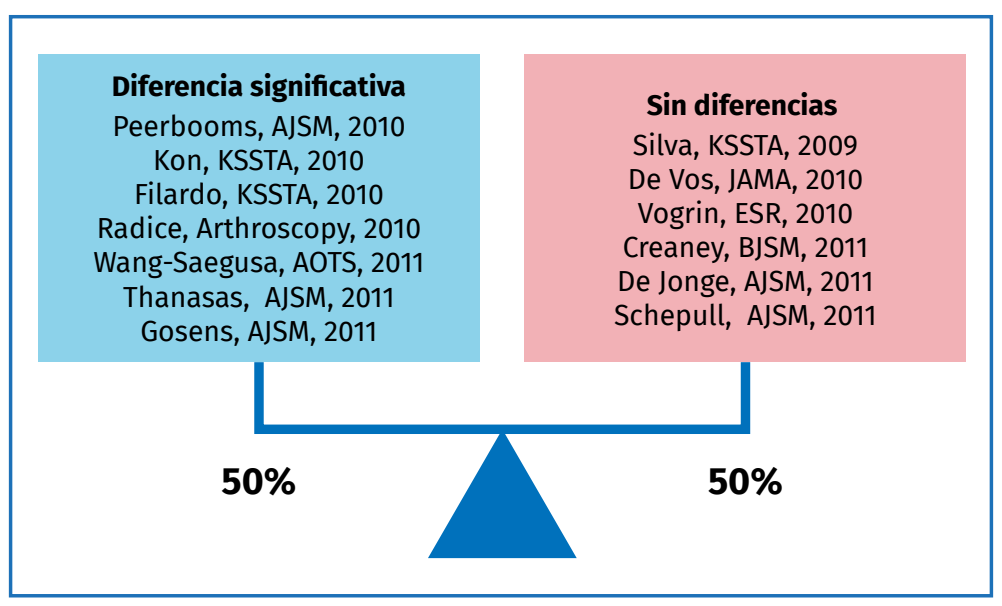

Figura 1. Evidencia en el uso de plasma rico en plaquetas (PRP).
Las dificultades para conseguir evidencias científicas claras son enormes por la cantidad de variables y potenciales sesgos (Figura 1).

\section{La evidencia científica en el plasma rico en plaquetas}

\section{En el sistema musculoesquelético en general}

La MBE en distintas áreas de interés para el cirujano ortopédico y traumatólogo en el campo de los PRP es poco concluyente hasta hoy.

Hay pocos estudios en esta mateestimular la proliferación de progenitores osteoblásticos. EL TGF- $\beta$ incrementa la sintesis de matriz y colágeno de tipo I. Los factores angiogénicos, como VEGF y FGF-2, pueden potencialmente favorecer la angiogénesis y la revascularización, como sugieren algunos trabajos ${ }^{(17)}$.

Todos estos factores los encontramos de forma natural en nuestro organismo y participan durante el proceso fisiológico de curación que la naturaleza ha desarrollado durante millones de años de evolución. De ahí que surja la pregunta de si podemos mejorar este proceso, sobre todo en el escenario de la lesión aguda, mediante el enriquecimiento de un ambiente ya de por sí rico.

El desarrollo del PRP se centra en potenciar este rico ambiente de curación natural. Las cuestiones claves sobre el PRP son: ¿pueden los PRP, en sus distintas variantes, mejorar este proceso de curación natural aumentando la concentración de FC? Y, si así fuera, ¿esta acción sería igual para potenciar la curación en distintos tejidos como el hueso o el tendón?

Varias publicaciones han defendido que el uso de FC en el tejido conectivo resulta en un aumento de la proliferación celular y una mayor proporción de matriz celular. Algunos estudios en animales han evaluado el efecto de PRP en lesiones musculares, publicando resultados de una mejor reparación celular y una menor fibrosis tisular.

Pero estos resultados preclínicos y en el campo de las ciencias básicas no han podido refrendarse en la clínica en estudios de alta evidencia que los dote de un alto grado de recomendación. ria con un nivel I o II de evidencia científica (EC), debido sin duda a la dificultad logística y ética de realizar estudios aleatorizados en este campo o de conseguir un número suficiente de casos para conformar cohortes numerosas que, seguidas en estudios prospectivos bien diseñados, pudieran resultar herramientas sólidas para dicha validación.

Los metaanálisis más actuales continúan concluyendo que no existe suficiente evidencia estadística, si bien todos ellos apuntan hacia la idea de que se necesita una estandarización de los métodos de preparación del PRP, como ya apuntamos en apartados anteriores ${ }^{(18)}$.

En una revisión sistemática y metaanálisis sobre los PRP en ortopedia de Sheth et al.(19), buscando determinar la eficacia del PRP en la disminución del dolor, mejora de la curación y mejora funcional, concluyeron que la evidencia es incierta y débil. Lo atribuyen muy directamente a la falta de estandarización de los protocolos de estudio, las técnicas de obtención del PRP y la medida de resultados.

\section{En tendinopatías}

La evidencia de los PRP en tendinopatías sigue siendo poco concluyente, con artículos que reportan mejoría y otros que reportan diferencias no significativas (Tabla 2 ).

En el metaanálisis de Andia et al. ${ }^{(20)}$, no se encontró correlación entre distintas concentraciones de plaquetas y resultados ${ }^{(21-31)}$. 


\begin{tabular}{|c|c|c|c|c|}
\hline ESTUDIO & DIAGNÓSTICO & TIPO DE PRP & ESCALAS & RESULTADOS \\
\hline $\begin{array}{l}\text { Vetrano } \\
\text { AJSM,2013 }\end{array}$ & Tendinopatía rotuliana & LR-PRP & VAS, VISA-P & $\begin{array}{l}\text { Diferencias significativas en VAS, en VISA a } \\
6 \text { meses } \\
\text { En todos los parámetros a los } 12 \text { meses }\end{array}$ \\
\hline $\begin{array}{l}\text { Dragoo } \\
\text { AJSM,2014 }\end{array}$ & Tendinopatía rotuliana & LR-PRP & $\begin{array}{l}\text { VAS, VISA, } \\
\text { TEGNER, } \\
\text { LYSHOLM, SF-12 }\end{array}$ & $\begin{array}{l}\text { VISA: a) mejoría a los } 12 \text { meses; b) sin } \\
\text { diferencias a los } 24 \text { meses } \\
\text { LYSHOLM: sin diferencias }\end{array}$ \\
\hline $\begin{array}{l}\text { Schepull } \\
\text { AJSM,2011 }\end{array}$ & Rotura tendón de Aquiles & LR-PRP & $\begin{array}{l}\text { Achilles Tendon } \\
\text { Total Rupture } \\
\text { Score }\end{array}$ & No diferencias significativas (vs. control) \\
\hline $\begin{array}{l}\text { De Jonge } \\
\text { AJSM,2011 }\end{array}$ & Tendinopatía de Aquiles & $\begin{array}{l}\text { LR-PRP } \\
\text { (GPS }{ }^{\circledR} \text { III, } \\
\text { Biomet) }\end{array}$ & VAS, VISA-A & $\begin{array}{l}\text { No diferencias significativas (vs. suero } \\
\text { fisiológico) }\end{array}$ \\
\hline $\begin{array}{l}\text { De Vos } \\
\text { JAMA, } 2010\end{array}$ & Tendinopatía de Aquiles & LP-PRP & VAS, VISA-A & $\begin{array}{l}\text { No diferencias significativas } \\
\text { (vs. suero fisiológico) }\end{array}$ \\
\hline $\begin{array}{l}\text { Krogh } \\
\text { AJSM,2013 }\end{array}$ & Tendinopatía del codo & LP-PRP & PRTEE & $\begin{array}{l}\text { No diferencias significativas a los } 3 \text { meses } \\
\text { (vs. suero y esteroides) }\end{array}$ \\
\hline $\begin{array}{l}\text { Creaney } \\
\text { AJSM,2011 }\end{array}$ & Tendinopatía del codo & LP-PRP & PRTEE & $\begin{array}{l}\text { No diferencias significativas } \\
\text { (vs. sangre autóloga pura) }\end{array}$ \\
\hline $\begin{array}{l}\text { Kesikburun } \\
\text { BJSM,2013 }\end{array}$ & $\begin{array}{l}\text { Tendinopatía del } \\
\text { manguito rotador }\end{array}$ & $\begin{array}{l}\text { LR-PRP } \\
\text { (GPS }{ }^{\circledR} \text { III, } \\
\text { Biomet) }\end{array}$ & WORC, SPADI, VAS & $\begin{array}{l}\text { No diferencias significativas al año } \\
\text { (vs. placebo) }\end{array}$ \\
\hline $\begin{array}{l}\text { Raeissadat } \\
\text { PRT,2014 }\end{array}$ & Tendinopatía del codo & LR-PRP & VAS, PPT, Mayo & $\begin{array}{l}\text { No diferencias significativas } \\
\text { (vs. sangre autóloga) }\end{array}$ \\
\hline $\begin{array}{l}\text { Mishra } \\
\text { AJSM,2014 }\end{array}$ & Tendinopatía del codo & $\begin{array}{l}\text { LR-PRP } \\
\text { (GPS }{ }^{\circledR} \text { III, } \\
\text { Biomet) }\end{array}$ & VAS & $\begin{array}{l}\text { No diferencias a las } 12 \text { semanas } \\
\text { Mejoría escalas clínicas a las } 24 \text { semanas } \\
\text { (vs. control) }\end{array}$ \\
\hline $\begin{array}{l}\text { Thanasas } \\
\text { AJSM,2011 }\end{array}$ & Tendinopatía del codo & $\begin{array}{l}\text { LR-PRP } \\
\text { (GPS }{ }^{\circledR} \text { III, } \\
\text { Biomet) }\end{array}$ & VAS, Liverpool & $\begin{array}{l}\text { Mejoría significativa en el VAS a las } 6 \\
\text { semanas (vs. sangre autóloga pura) }\end{array}$ \\
\hline $\begin{array}{l}\text { Peerbooms } \\
\text { AJSM,2010 }\end{array}$ & Tendinopatía del codo & $\begin{array}{l}\text { LR-PRP } \\
\text { (GPS }{ }^{\circledR} \text { III, } \\
\text { Biomet) }\end{array}$ & VAS, DASH & $\begin{array}{l}\text { Mejoría significativa en VAS y DASH } \\
\text { (vs. corticoesteroides) }\end{array}$ \\
\hline
\end{tabular}

En el metaanálisis de Tsikopoulos et al. ${ }^{(32)}$ también concluyen que el PRP no produjo beneficios clínicos significativos, en comparación con placebo o punción seca en el tratamiento de las tendinopatías en general, con la salvedad del manguito rotador, donde sí se encuentra una leve diferencia.

En el último metaanálisis de Cochrane ${ }^{(18)}$ publicado en 2014 sobre el uso de PRP en lesiones musculoesqueléticas sobre tejidos blandos, los autores concluyen que la evidencia era insuficiente para aconsejar el uso de PRP para lesiones de tejidos blandos en general. No obstante, ya se apunta la necesidad de estandarizar los métodos de preparación y generar formulaciones específicas.

Por otro lado, en un reciente metaanálisis de

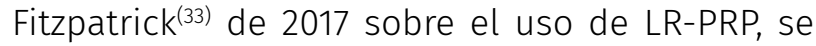
concluye que hay una buena evidencia que apoya el uso de una inyección aislada de LR-PRP bajo control ecográfico en tendinopatías. Se recalca específicamente que tanto la inyección intratendinosa como la preparación del LR-PRP son de gran relevancia. 


\section{En tendinopatía de Aquiles}

La tendinopatía no insercional del Aquiles puede ser tratada con distintas terapias. Cuando las medidas conservadoras fracasan, se han propuesto alternativas, entre las que se encuentra el tratamiento con PRP.

En los escasos ensayos clínicos controlados publicados a fecha de hoy, los datos no han mostrado mejorías clínicas estadísticamente significativas con la inyección de PRP. Kearney et al. ${ }^{(34)}$ condujeron un estudio piloto aleatorizando los pacientes para recibir tratamiento con PRP versus un programa de ejercicios excéntricos de Aquiles. No se encontraron diferencias estadísticas entre ambos grupos.

De forma similar, De Vos et al. ${ }^{(35,36)}$ condujeron un estudio aleatorizado doble ciego comparando pacientes en tratamiento con excéntricos de Aquiles y uso de PRP con pacientes en tratamiento con excéntricos de Aquiles y suero fisiológico. Los pacientes mejoraron en ambos grupos pero sin diferencias significativas entre ambos.

Podría concluirse que, en la tendinopatía no insercional de Aquiles, la EC actual no apoya de forma clara el uso de PRP, dado que no se han encontrado en los distintos trabajos beneficios adicionales a las terapias convencionales. Sin embargo, estudios como el previamente mencionado metaanálisis de Fitzpatrick en tendinopatías en general y considerando específicamente la formulación del PRP como LR-PRP nos ponen en la pista de nuevos caminos para profundizar en la búsqueda de evidencias científicas en este campo, para esta y otras indicaciones.

\section{En heridas crónicas}

Existen diversos trabajos en relación con el papel de los PRP sobre la curación de las úlceras y heridas crónicas, con especial interés en las úlceras asociadas a la diabetes. No obstante, no se conoce con claridad la influencia y el mecanismo del PRP sobre las úlceras crónicas. La calidad de la EC del PRP sobre las úlceras crónicas es baja.

En un metaanálisis de Cochrane de 2016 los autores concluyen que el PRP puede mejorar la curación de las úlceras en diabéticos, sin bien esta conclusión está sustentada en evidencias de baja cualidad dadas por 2 pequeños ensayos clínicos controlados aleatorizados ${ }^{(37)}$. Estos estudios no son por ello suficientemente potentes, ni con diseños capaces de evitar riesgo de sesgos. Por tanto, se concluye que se necesitan ensayos clínicos mejor diseñados para extraer conclusiones más definitivas.

\section{En artrosis}

Los resultados en la literatura sobre el uso del PRP intraarticular en el tratamiento de la artrosis son dispares.

Se debate sobre la importancia de la presencia de células blancas y células rojas en el efecto final de las preparados de PRP. Algunos estudios han mostrado el efecto de distintas formulaciones de PRP sobre los sinoviocitos. Así, se ha visto que la presencia de células rojas genera una muerte celular de sinoviocitos mucho mayor. Las formulaciones ricas en leucocitos (LR-PRP) también generan mayor muerte de sinoviocitos ${ }^{(38)}$.

El mejor perfil para la viabilidad de los sinoviocitos se obtiene con PRP pobres en leucocitos (LP-PRP) $^{(39)}$ (Tabla 3).

El artículo de Riboh et al. ${ }^{(40)}$ concluye que el LPPRP mejora los parámetros funcionales en comparación con ácido hialurónico y placebo en casos de artrosis de rodilla (Tabla 4).

\section{En lesiones musculares}

Las publicaciones en lesiones miofibrilares agudas son pobres. En un metaanálisis de Pas et al. ${ }^{(41)}$, se concluye que no existe evidencia para el uso de PRP sobre lesiones agudas en isquiotibiales, existiendo numerosas limitaciones en el diseño de los estudios analizados.

\section{En regeneración ósea}

El papel que puede jugar el uso de PRP en la reparación ósea es todavía controvertido. La capacidad de promover o inhibir la formación ósea, en función de la calidad y composición del PRP, requiere todavía mejores estudios para definir el rol de estos tratamientos como adyuvancia al uso de injertos óseos ${ }^{(42)}$. 


\begin{tabular}{|c|c|c|c|c|c|}
\hline $\begin{array}{l}\text { TIPO DE } \\
\text { ESTUDIO }\end{array}$ & AUTOR & DIAGNÓSTICO & $\begin{array}{l}\text { COMPOSICIÓN } \\
\text { PRP }\end{array}$ & ESCALAS & RESULTADOS \\
\hline $\begin{array}{l}\text { Doble ciego } \\
\text { aleatorizado } \\
(n=78)\end{array}$ & Patel, AJSM, 2013 & $\begin{array}{l}\text { Osteoartrosis } \\
\text { bilateral de } \\
\text { rodilla }\end{array}$ & LP-PRP & $\begin{array}{l}\text { WOMAC } \\
\text { VAS }\end{array}$ & $\begin{array}{l}\text { Diferencia significativa } \\
\text { (vs. suero) }\end{array}$ \\
\hline $\begin{array}{l}\text { Doble ciego } \\
\text { aleatorizado } \\
(n=109)\end{array}$ & $\begin{array}{l}\text { Filardo, } \\
\text { BMC Musculoskel Disord } \\
2012\end{array}$ & $\begin{array}{l}\text { Osteoartrosis } \\
\text { grados } 1 \text { a } 3\end{array}$ & LP-PRP & $\begin{array}{l}\text { AIKDC, EQ-VAS, } \\
\text { Tegner, KOOS }\end{array}$ & Sin diferencia (vs. AH) \\
\hline $\begin{array}{l}\text { Aleatorizado } \\
(n=120)\end{array}$ & Cerza, AJSM, 2012 & $\begin{array}{l}\text { Osteoartrosis } \\
\text { grados } 1 \text { a } 3\end{array}$ & LP-PRP & WOMAC & $\begin{array}{l}\text { Diferencia significativa } \\
\text { (vs. } \mathrm{AH} \text { ) }\end{array}$ \\
\hline $\begin{array}{l}\text { Doble ciego } \\
\text { aleatorizado } \\
(n=176)\end{array}$ & $\begin{array}{l}\text { Sanchez, Arthroscopy, } \\
2012\end{array}$ & $\begin{array}{l}\text { Osteoartrosis } \\
\text { de rodilla }\end{array}$ & PRGF, LP-PRP & $\begin{array}{l}\text { Knee pain } \\
\text { WOMAC }\end{array}$ & $\begin{array}{l}\text { Diferencia significativa } \\
\text { (vs. } \mathrm{AH} \text { ) }\end{array}$ \\
\hline $\begin{array}{l}\text { Cohorte } \\
\text { prospectiva } \\
(n=120)\end{array}$ & $\begin{array}{l}\text { Spakova, Am J Phys Med } \\
\text { Rehab, } 2012\end{array}$ & $\begin{array}{l}\text { Osteoartrosis } \\
\text { grados } 1 \text { a } 3\end{array}$ & LP-PRP & $\begin{array}{l}\text { WOMAC, } \\
\text { Numeric Rating } \\
\text { Scale }\end{array}$ & $\begin{array}{l}\text { Diferencia significativa } \\
\text { (vs. AH) }\end{array}$ \\
\hline $\begin{array}{l}\text { Cohorte } \\
\text { prospectiva } \\
(n=144)\end{array}$ & Filardo, KSSTA, 2012 & $\begin{array}{l}\text { Osteoartrosis } \\
\text { grados } 1 \text { a } 3\end{array}$ & PRGF vs. LR-PRP & $\begin{array}{l}\text { IKDC, Eq-VAS, } \\
\text { Tegner }\end{array}$ & $\begin{array}{l}\text { Más dolor e inflamación } \\
\text { con PRP }\end{array}$ \\
\hline
\end{tabular}

AH: ácido hialurónico; LP-PRP: plasma rico en plaquetas y rico en leucocitos; PRGF: preparado rico en factores de crecimiento

\section{Tabla 4. EVIDENCIA DEL USO DE PLASMA RICO EN PLAQUETAS (PRP) EN OSTEOARTROSIS DE RODILLA}

\section{USO DE PRP EN OSTEOARTROSIS DE RODILLA}

\section{Metaanálisis/Revisiones}

- 2016, Journal of Arthroscopy: PRP mejores resultados vs. ácido hialurónico (AH) a los 12 meses

\section{Nivel 1 o 2 de evidencia}

-2016, Int J Mol Sci: PRP mejores resultados vs. AH a los 3 meses y mejores resultados en osteoartrosis de bajo grado

- 2016, AJSM: LP-PRP (ACP) disminución de escala WOMAC al año de un 78 vs. $7 \%$ placebo

- 2016, Ar North Trauma Sx: LP-PRP mejores resultados vs. acetaminofeno a las 24 semanas

- 2016, Jsp Med Ph Fil: PRP mejores resultados vs. corticosteroides en escalas de dolor y funcionalidad a los 6 meses

- 2016, Knee SST Arth: en osteoartrosis moderadas-avanzadas, PRP mejores resultados vs. AH y ozonoterapia a los 12 meses

\section{Conclusiones}

El PRP ha ido introduciéndose en el manejo terapéutico con una enorme variabilidad en las técnicas de obtención y en las pautas utilizadas. Por tanto, no es de sorprender que el nivel de EC que lo acompaña sea bajo, lo que no ha de impedir que sea utilizado en pacientes y situaciones bien seleccionados.

Los metaanálisis más actuales continúan concluyendo que no existe suficiente evidencia, si bien todos apuntan hacia la idea de que se necesita una estandarización de los métodos de preparación del PRP y formulaciones específicas ${ }^{(43)}$.

La gran mayoría de los estudios y revisiones sistémicas obvian una información que en los últimos años parece crucial para entender el papel de los PRP: su formulación y composición específica. Así, los últimos estudios apuntan hacia la obtención de preparados específicos con la concentración óptima para cada indicación de FC, plaquetas y leucocitos, así como su activación o no, o la adición de fibrina. 
La información actual respecto al binomio formulación-indicación orienta a utilizar PRP sin células blancas ni eritrocitos (LP-PRP) para su uso intraarticular, mientras que aconsejaría incluir células blancas (LR-PRP) en procesos como las tendinopatías crónicas.

Las guías clínicas, basadas en la evidencia, se beneficiarán sin duda de estudios más homogéneos y reproducibles.

\section{Bibliografía}

1. Hsu WK, Mishra A, Rodeo SR, Fu F, Terry MA, Randelli P, et al. Platelet-rich plasma in orthopaedic applications: evidence-based recommendations for treatment. J Am Acad Orthop Surg. 2013;21(12):739-48.

2. Marx RE. Platelet-rich plasma: evidence to support its use. J Oral Maxillofac Surg. 2004;62(4):489-96.

3. Weibrich G, Kleis WK, Hitzler WE, Hafner G. Comparison of the platelet concentrate collection system with the plasma-rich-in-growth-factors kit to produce platelet-rich plasma: A technical report. Int J Oral Maxillofac Implants. 2005;20:118-23

4. Reyes M. Actualización de la Técnica de Obtención y Uso del Plasma Rico en Factores de Crecimiento (PRGF). Rev Dent Chile. 2002;93(2):25-8.

5. Wroblewski AP, Melia HJ, Wright VJ. Application of platelet-rich plasma to enhance tissue repair. Oper Tech Orthop. 2010;20:98-105.

6. Ley $29 / 2006$, de 26 de julio, de garantías y uso racional de los medicamentos y productos sanitarios. Jefatura del Estado. BOE núm. 178, de 27 de julio de 2006, pp. 28122-65.

7. Spaova T, Rosita J, Lack M, Harvanová D, Gharaibeh A. Treatment of knee joint osteoarthritis with autologous platelet-rich plasma in comparison with hyaluronic acid. Am J Phys Med Rehabil Assoc Acad Physiatr. 2012;91(5):411-7.

8. Moreno, R., Gaspar M., Jiménez J., Alonso JM., Villimar A, López P. Técnicas de obtención del plasma rico en plaquetas y su empleo en terapéutica osteoinductora. Farm Hosp. 2015;39(3):130-6.

9. Dohan Ehrenfest DM, Andia I, Zumstein MA, Zhang C, Pinto NR, Bielecki T. Classification of platelet concentrates (Platelet-Rich Plasma-PRP, Platelet-Rich Fibrin-PRF) for topical and infiltrative use in orthopedic and sports medicine: current consensus, clinical implications and perspectives. Muscles Ligaments Tendons J. 2014;4(1):3-9.

10. Sundman EA, Cole BJ, Fortier LA. Growth factor and catabolic cytokine concentrations are influenced by the cellular composition of platelet-rich plasma. Am J Sports Med. 2011;39(10):2135-40.

11. Dragoo JL, Braun HJ, Durham JL, Ridley BA, Odegaard JI, Luong R, Arnoczky SP. Comparison of the acute inflamatory response of two comercial PRP system in healthy rabbit tendons. Am J Sports Med. 2012;40(6):1274-81.

12. Directiva 2001/83/CE del Parlamento Europeo y del Consejo, de 6 de noviembre de 2001, por la que se establece un código comunitario sobre medicamentos para uso humano. Comunidades europeas. DOUE núm. 311, de 28 de noviembre de 2001, pp. 67-128.

13. Real Decreto $1591 / 2009$, de 16 de octubre, por el que se regulan los productos sanitarios. Ministerio de Sanidad y Política Social. BOE núm. 268, de 6 de noviembre de 2009, pp. 92708-78.

14. Guidelines relating to the application of the Council Directive 93/42/EEC on medical devices. European Commission DG Health and Consumer. Directorate B, Unit B2 "Cosmetics and medical devices". MEDDEV 2. 4/1 Rev. 9 June 2010. Disponible en: http://ec.europa. eu/health/medical-devices/files/meddev/2_4_1_ rev_9_ classification.

15. De La Mata J. Plasma rico en plaquetas: ¿un nuevo tratamiento para el reumatólogo? Reumatol Clínica. 2013;9(3):166-71.

16. Peidro L, Segur JM, Poggio D, Fernandez de Retana P. Use of freeze-dried bone allograft with platelet-derived growth factor for revision of a glenoid component. JBJS Br. 2006;88-B:9.

17. Rai B, Oest ME, Dupont KM, Ho KH, Teoh SH, Guldberg RE. Combination of platelet-rich plasma with polycaprolactone-tricalcium phosphate scaffolds for segmental bone defect repair. J Biomed Mater Res A. 2007;81:888-99.

18. Moraes VY, Lenza M, Tamaoki MJ, Faloppa F, Belloti JC. Platelet-rich therapies for musculoskeletal soft tissue injuries. Cochrane Database Syst Rev. 2014;(4).

19. Sheth U, Simunovic N, Klein G, Fu F, Einhorn TA, Schemitsch E, et al. Efficacy of autologous platelet-rich plasma use for orthopaedic indications: a meta-analysis. J Bone Joint Surg Am. 2012;94(4):298-307.

20. Andia I, Latorre PM, Gomez MC, Burgos-Alonso N, Abate $M$, Mafulli N. Platelet-rich plasma in the conservative treatment of painful tendinopathy: a systematic review and meta-analysis of controlled studies. Br Med Bull. 2014;110(1):99-115.

21. Creaney L, Walla A, Curtis M, Connell D. Growth factor-based therapies provide additional benefit beyond physical therapy in resistant elbow tendinopathy: a prospective, double-blind, randomised trial of autol- 
ogous blood injec- tions versus platelet-rich plasma injections. Br J Sports Med. 2011;45:966-71.

22. De Vos RJ, Weir A, van Schie HT, Bierma-Zeinstra SM, Verhaar JA, Weinans $H$, Tol JL. Platelet-rich plasma injection for chronic Achilles tendinopathy: a randomized controlled trial. JAMA. 2010;303:144-9.

23. Dragoo JL, Wasterlain AS, Braun HJ, Nead KT. Platelet-rich plasma as a treatment for patellar tendinopathy: a double-blind, randomized controlled trial. Am J Sports Med. 2014;42:610-8.

24. Filardo G, Kon E, Della Villa S, Vincentelli F, Fornasari PM, Marcacci M. Use of platelet-rich plasma for the treatment of refractory jumper's knee. Int Orthop. 2010;34:909-15.

25. Kesikburun S, Tan AK, Yilmaz B, Yaşar E, Yazicioğlu K. Platelet-rich plasma injections in the treatment of chronic rotator cuff tendinopathy: a randomized controlled trial with 1-year follow-up. Am J Sports Med. 2013;41:2609-16.

26. Krogh TP, Fredberg U, Stengaard-Pedersen K, Christensen $\mathrm{R}$, Jensen $\mathrm{P}$, Ellingsen $\mathrm{T}$. Treatment of lateral epicondylitis with platelet-rich plasma, glucocorticoid, or saline: a randomized, double-blind, placebo-controlled trial. Am J Sports Med. 2013;41:625-35.

27. Mishra AK, Skrepnik NV, Edwards SG, Jones GL, Sampson S, Vermillion DA, et al. Platelet-rich plasma significantly improves clinical outcomes in patients with chronic tennis elbow: a double-blind, prospective, multicenter, controlled trial of 230 patients. Am J Sports Med. 2014;42:463-71.

28. Peerbooms JC, Sluimer J, Bruijn DJ, Gosens T. Positive effect of an autologous platelet concentrate in lateral epicondylitis in a double-blind randomized controlled trial: platelet-rich plasma versus corticosteroid injection with a 1-year follow-up. Am J Sports Med. 2010;38:255-62.

29. Raeissadat SA, Rayegani SM, Hassanabadi H, Rahimi R, Sedighipour L, Rostami K. Is platelet-rich plasma superior to whole blood in the management of chronic tennis elbow: one year randomized clinical trial. BMC Sports Sci Med Rehabil. 2014;6:12.

30. Thanasas C, Papadimitriou G, Charalambidis C, Paraskevopoulos I, Papanikolaou A. Platelet-rich plasma versus autologous whole blood for the treatment of chronic lateral elbow epicondylitis: a randomized controlled clinical trial. Am J Sports Med. 2011;39:2130-4.

31. Omar Aziza S, Maha El, Amal SA, Mahmoud S. Local injection of autologous platelet rich plasma and corticosteroid in treat- ment of lateral epicondylitis and plantar fasciitis: randomized clinical trial. Egypt Rheumatol. 2012;34:43-9.
32. Tsikopoulos K, Tsikopoulos I, Simeonidis E, Papathanasiou E, Haidich AB, Anastasopoulos N, Natsis K. The clinical impact of platelet-rich plasma on tendinopathy compared to placebo or dry needling injections: a meta-analysis. Phys Ther Sport. 2016;17:87-94.

33. Fitzpatrick J, Bulsara M, Zheng MH. The Effectiveness of Platelet-Rich Plasma in the Treatment of Tendinopathy. Am J Sports Med. 2017;45(1):226-33.

34. Kearney RS, Parsons N, Costa ML. Achilles tendinopathy management: a pilot randomised controlled trial comparing platelet-rich plasma injection with an eccentric loading programme. Bone Joint Res. 2013;2(10):227-32.

35. De Vos RJ, Weir A, Tol JL, Verhaar JA, Weinans H, van Schie HT. No effects of PRP on ultrasonographic tendon structure and neovascularisation in chronic midportion Achilles tendinopathy. Br J Sports Med. 2011;45(5):387-92.

36. De Vos RJ, Weir A, van Schie HT, Bierma-Zeinstra SM, Verhaar JA, Weinans H, Tol JL. Platelet-rich plasma injection for chronic Achilles tendinopathy: a randomized controlled trial. JAMA. 2010;303(2):144-9.

37. Martinez-Zapata MJ, Martí-Carvajal AJ, Solà I, Expósito JA, Bolíbar I, Rodríguez L, et al. Autologous platelet-rich plasma for treating chronic wounds. Cochrane Database Syst Rev. 2016;(5).

38. Schneider BS, Tiidus PM. Neutrophil infiltration in exercise-injured skeletal muscle: how do we resolve the controversy? Sports Med. 2007;37(10):837-56.

39. Braun HJ, Kim HJ, Chu CR, Dragoo JL. The effect of platelet-rich plasma formulations and blood products on human synoviocytes: implications for intrarticular injury and therapy. Am J Sports Med. 2014;42(5):1204-10.

40. Riboh JC, Saltzman BM, Yanke AB, Fortier L, Cole BJ. Effect of Leukocyte Concentration on the Efficacy of Platelet-Rich Plasma in the Treatment of Knee Osteoarthritis. Am J Sports Med. 2016;44(3):792-800.

41. Pas HI, Reurink G, Tol JL, Weir A, Winters M, Moen MH. Efficacy of rehabilitation (lengthening) exercises, platelet-rich plasma injections, and other conservative interventions in acute hamstring injuries: an updated systematic review and meta-analysis. Br J Sports Med. 2015;49(18):1197-205.

42. Muschler GF, Matsukura $Y$, Nitto H, Boehm CA, Valdevit $A D$, Kambic HE, et al. Selective retention of bone marrow-derived cells to enhance spinal fusion. Clin Orthop Relat Res. 2005;(432):242-51.

43. Sheth U, Simunovic N, Klein G, Fu F, Einhorn TA, Schemitsch E, et al. Efficacy of Autologous Platelet-Rich Plasma Use for Orthopaedic Indications: A Meta-Analysis. J Bone Joint Surg Am. 2012;94(4):298-307. 